\title{
Phase-space networks of the six-vertex model under different boundary conditions
}

\author{
Yilong Han (韩一龙) \\ Department of Physics, Hong Kong University of Science and Technology, Clear Water Bay, Kowloon, Hong Kong, China
}

(Received 17 December 2009; published 16 April 2010; corrected 27 April 2010)

\begin{abstract}
The six-vertex model is mapped to three-dimensional sphere stacks and different boundary conditions corresponding to different containers. The shape of the container provides a qualitative visualization of the boundary effect. Based on the sphere-stacking picture, we map the phase spaces of the six-vertex models to discrete networks. A node in the network represents a state of the system, and an edge between two nodes represents a zero-energy spin flip, which corresponds to adding or removing a sphere. The network analysis shows that the phase spaces of systems with different boundary conditions share some common features. We derived a few formulas for the number and the sizes of the disconnected phase-space subnetworks under the periodic boundary conditions. The sphere stacking provides new challenges in combinatorics and may cast light on some two-dimensional models.
\end{abstract}

DOI: 10.1103/PhysRevE.81.041118

PACS number(s): 05.50.+q, 64.60.aq, 75.10.Hk

\section{INTRODUCTION}

Phase space structures are important for understanding the dynamics of the system. However they are usually too large and complicated to be analyzed quantitatively. Here, we map the phase spaces of a spin model into complex networks so that quantitative network analysis $[1-3]$ can be applied to the phase-space study. Spins on lattices have discrete degrees of freedom, such that their phase spaces are discrete and can be viewed as networks. One node in the network corresponds to one configuration state of the system. Two nodes are connected by an edge (or link) if the system can directly evolve from one state to the other without passing through intermediate states. Edges are undirected because dynamic processes at the microscopic level are time reversible. In [4], we constructed the ground-state phase-space networks of two classical frustrated spin models, namely, antiferromagnet on triangular lattice and square ice. Their phase spaces share some common features, including the small-world property, Gaussian-like connectivity distributions and Gaussian spectral densities. Here we showed that these features also exist in the phase spaces of the six-vertex models $[5,6]$ under different boundary conditions. Note that the square ice is a special case of the six-vertex model in a square area with the domain-wall boundary condition.

To construct the phase-space network, we first generate all the possible states of the spin system, i.e., all the nodes in the network. The more challenging step is to identify whether two nodes are connected by an edge. A convenient way to construct the phase-space network is to map the spin model to a higher dimensional stack so that adding or removing a building block of the stack corresponds to an edge in the network [4]. For example, in the elegant one-to-one mapping between the two-dimensional (2D) antiferromagnet on triangular lattice [7] and the three-dimensional (3D) cube stack [8], adding or removing a cube corresponds to a basic zeroenergy spin flip. Hence, two states are connected in the phase-space network if they are different by one cube [4]. Recently, we directly observed such zero-energy spin flips in a colloidal experiment [9]. Here we mapped the 2D sixvertex models to the 3D close-packed sphere stacks before the network construction. The sphere stacks also provide a direct visualization of the boundary effect and make the symmetries of the system more transparent. Moreover, the sphere stacking approach helped us to derive a few formulas for the number and the sizes of the disconnected subnetworks of the phase spaces under the periodic boundary conditions.

\section{MAPPING BETWEEN THE SIX-VERTEX MODEL AND SPHERE STACKS}

The six-vertex model is a spin ice on square lattice, see the example with the domain-wall boundary condition in Fig. 1(a). The arrows follow the ice rule, i.e., each vertex has two incoming and two outgoing arrows. Consequently each vertex has $C_{4}^{2}=6$ possible configurations as shown in Fig. 1(b). If the arrows are taken as ferromagnetic spins, geometrical frustration arises as shown in Fig. 1(c). Flipping a closed loop of arrows from clockwise to counterclockwise (or vice versa) does not break the ice rule and costs no energy. The smallest local zero-energy mode is the flipping of the fourarrow loop shown in Fig. 1(d). We call such flipping the basic flip. [5] shows that any configuration change can be decomposed into a sequence of such flips under a fixed boundary condition.

The spin ice configuration in Fig. 1(a) has one-to-one correspondences to the square ice shown in Fig. 1(f), and the jigsaw tiling and alternating signed matrix shown in Fig. 1(g) [10]. The alternating signed matrixes are square matrixes with entries 0 or \pm 1 such that each row and column has an alternating sequence of +1 and -1 (zeroes excluded) starting and ending with +1 . In combinatorics, the number of $L \times L$ alternating signed matrixes is [10]

$$
\begin{aligned}
N_{n}(L) & =\prod_{1 \leq i \leq j \leq L} \frac{L+i+j-1}{2 i+j-1}=\prod_{j=0}^{L-1} \frac{(3 j+1) !}{(L+j) !} \\
& \sim\left(\frac{27}{16}\right)^{\left(L^{2} / 2\right)} \text { when } L \rightarrow \infty .
\end{aligned}
$$

This formula also gives the number of nodes in the phasespace network of the $L \times L$ spin ice under the domain-wall boundary condition. 


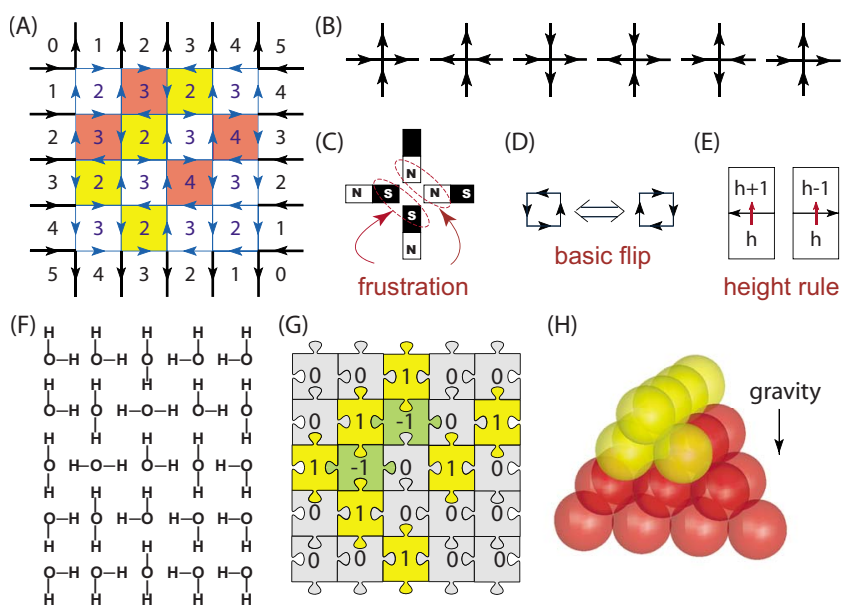

FIG. 1. (Color online) (a) A configuration of the six-vertex model (or the spin ice) on a $5 \times 5$ lattice with the domain-wall boundary condition. Each plaquette is assigned a height based on the height rule in (e). The upper left corner is defined as height zero. The basic flips are the four-arrow loops labeled in red (clockwise) and yellow (counterclockwise). (b) The six allowed types of vertexes that satisfy the ice rule. (c) Four magnets placed at a cross inevitably have frustrations. (d) The basic flip. (e): The height rule. $(\mathrm{f}-\mathrm{h})$ Three equivalent representations of (a). (f) Water molecules frozen on a square lattice, i.e., square ice. Hydrogen atoms correspond to outgoing arrows. (g) Each jigsaw tile [10] can be viewed as a water molecule with one oxygen atom in the center and two hydrogen atoms at the two bulges. By assigning vertical tiles as 1 , horizontal tiles as -1 and the other four types as 0 , a $5 \times 5$ alternating signed matrix [10] is obtained. (h) The corresponding red sphere stack, which is stable under gravity. Yellow spheres are vacant sites. The four removable red spheres and four addable yellow sites on the top of the red-sphere stack correspond to the four red and four yellow plaquettes in (a).

To construct the phase-space network, we first map the six-vertex model as sphere stacks in the face-centered cubic (FCC) lattice shown in Fig. 1(h), so that each basic flip is equivalent to adding or removing a sphere. In Fig. 1(a), each square plaquette is assigned a height based on the height rule [5] shown in Fig. 1(e): When walking from the plaquette with height $h$ to its neighbor, the height increases by 1 if it crosses a left arrow and decreases by 1 if it crosses a right arrow. The ice rule guarantees that the height change around a vertex is zero and the height is independent of the path along which it was computed. A plaquette can flip only when its four neighbor plaquettes have the same height. This indicates that each building block is "supported" by four blocks underneath in an effective "gravity field." Thus, the stack can be viewed as an FCC stacking along the [100] direction. An FCC lattice can be conveniently represented by a stack of close-packed spheres. This stacking is better visualized in Fig. 2. Figure 2(a) shows the lowest possible heights, which contain no clockwise four-arrow loop. These lowest heights define a vacant container made of two tilted triangles as shown in the lower panel of Fig. 2(a). Each basic flip changes the height by 2 , which equals to adding or removing a sphere of unit radius. Flipping all available counterclockwise loops (yellow plaquettes) is equivalent to adding a layer of spheres, see Figs. 2(b)-2(f). The maximum heights in Fig.

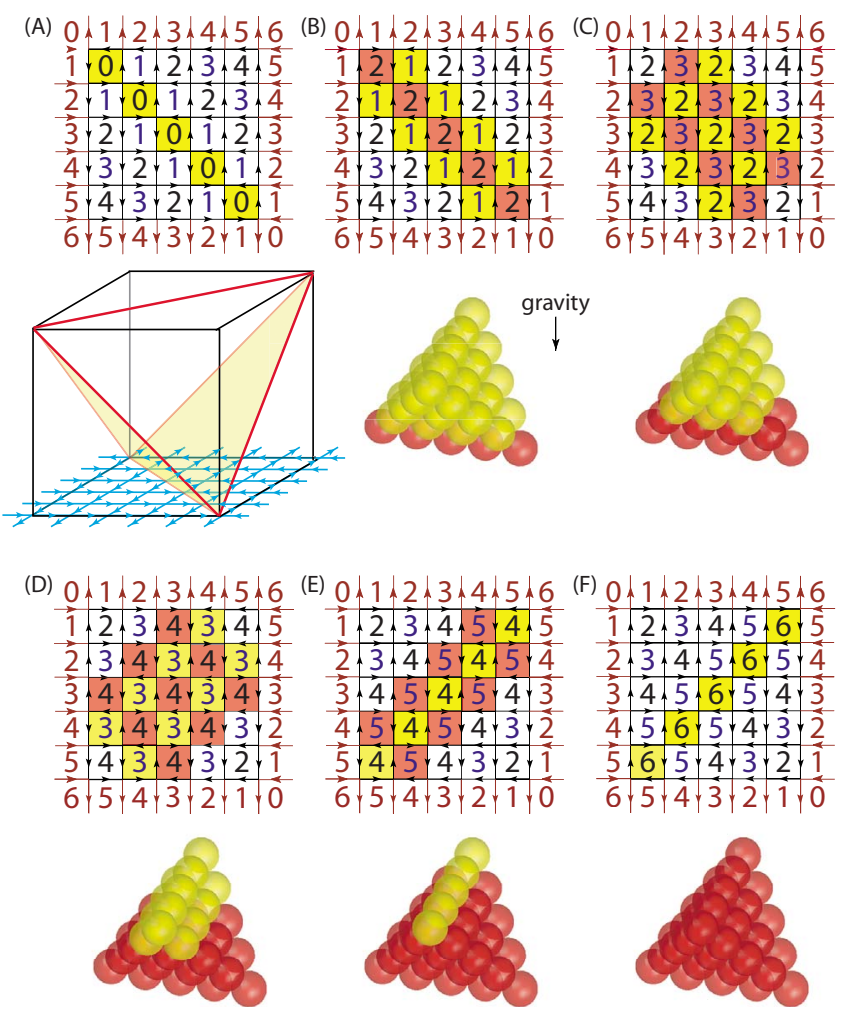

FIG. 2. (Color online) The one-to-one correspondence between the six-vertex models with domain-wall boundary conditions and sphere stacks in a tetrahedron. (a) The lowest possible heights under this boundary condition. The zero height is defined as the upper left plaquette and other heights are generated by the height rule in Fig. 1(e). These heights define the two yellow triangles in the bottom panel. (b) After flipping the five yellow counterclockwise four-spin loops in (a) to the red clockwise loops in (b), the heights of the five plaquettes are increased by 2 . It is equivalent to adding five red spheres as shown in the lower panel of (b). The yellow spheres are vacant sites. (c-f) Each figure is obtained by flipping all the yellow loops in the previous figure, i.e., adding a layer of spheres to the previous stack. The highest possible heights are shown in (f). These heights define a lid surface, which is an upside-down container in (a).

2(f) are reached by the continued flipping of counterclockwise loops to clockwise loops until no counterclockwise loop is left. The height of each plaquette represents the physical height of the corresponding sphere on the top surface of the stack. We generate all the legal configurations by flipping counterclockwise loops from the vacant state, i.e., adding spheres from the vacant container in Fig. 2(a). The heights in Fig. 2(f) define a lid made of anther two triangles. The container and the lid form a tetrahedron.

The six-vertex model has a similar solid-on-solid representation on the body-centered cubic (BCC) lattice [11]. Here we use the FCC sphere stacks because it appears to be a more natural representation with simple building-block shape and simple container shape. Moreover, FCC lattice can be represented by a simple polyhedra stacking under gravity, but BCC cannot. The Wigner-Seitz cell of the FCC lattice is a rhombic dodecahedron, which is supported by four rhombic dodecahedra underneath. In contrast, the Wigner-Seitz 

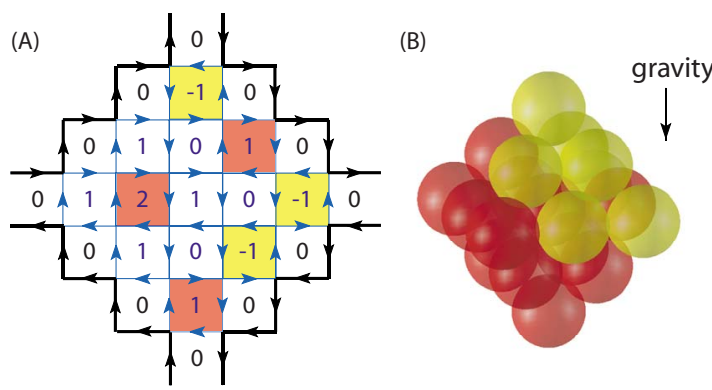

FIG. 3. (Color online) (a) A spin ice in a diamond area under the constant-height boundary condition. (b) The corresponding sphere stack in an octahedron. The yellow spheres are vacant sites.

cell of the BCC lattice has a flat square surface on the top so that it can be supported by only one block underneath. For a correct stacking, one building block should be supported by four blocks in order to reflect that one plaquette can flip only when the four neighbors have the same height.

\section{BOUNDARY EFFECTS}

The six-vertex model has been solved for the periodic [6], the anti-periodic [12] and the domain-wall boundary conditions [13]. We have shown that the domain-wall boundary condition corresponds to sphere stacks in a simple tetrahedron. We can easily design another simple boundary condition as shown in Fig. 3(a) which corresponds to sphere stacks in a simple octahedron. It would be interesting to explore whether this boundary condition can also be exactly solved in statistical mechanics [14].

Different boundary conditions correspond to different container shapes, for example, see Figs. 2-4. Given a fixed boundary condition, the lowest and the highest possible heights can be directly written out layer by layer from the boundary to the bulk, e.g., see Figs. 4(a) and 4(b). The colored plaquettes in Figs. 4(a) and 4(b) show that the lid contains one height maximum and the container contains nine height minima. These heights define a lid and a container with different shapes as shown in Figs. 4(d) and 4(e). The container and the lid form an interesting pair of dual surfaces: using one as the container, the other will emerge as the surface of the highest "sand pile" of small spheres, see Figs. 4(d), 4(e), 4(h), and 4(i). In fact, they are dual surfaces because packing spheres in the container is equivalent to packing buoyant spheres in the corresponding lid. The container and the lid would switch roles by reversing the height rule in Fig. 1(e). The height difference between the lid and the container in a square area appears to be a pyramid under all fixed boundary conditions shown in Figs. 3-5.

One peculiar property of frustrated spin models is that boundary effects often, but not always, percolate through the entire system even in the infinite-sized limit [15-17]. This can be directly visualized from the sphere stacks in containers. Figure 6(a) shows a typical sphere stack in an $L=100$ tetrahedron which has a central disordered region and four ordered frozen corners. The disordered "liquid" region contains basic flips, while the frozen areas do not. The averaged flipping probabilities of the $100 \times 100$ plaquettes are shown

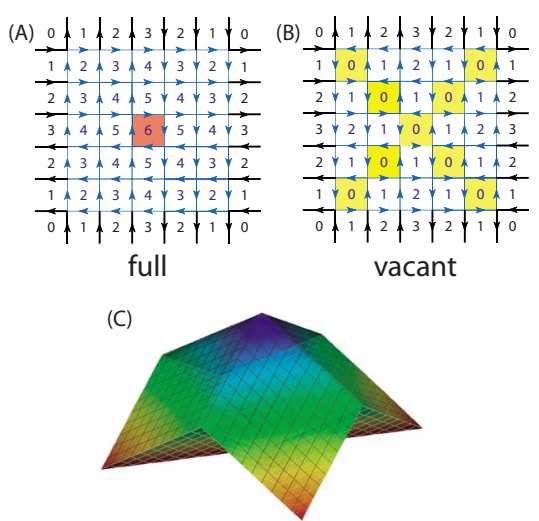

full packing
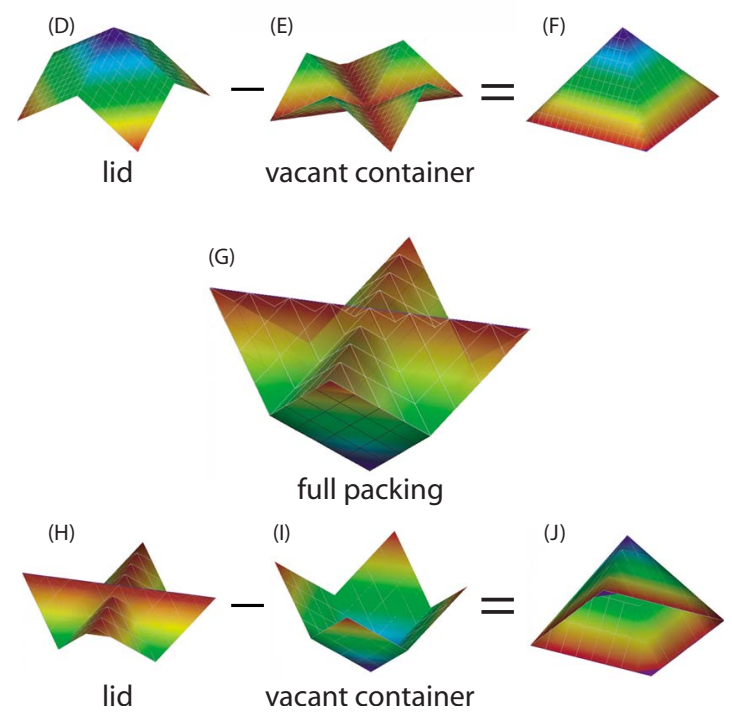

FIG. 4. (Color online) The lid-container duality of a $6 \times 6$ spin ice with the boundary condition shown in (a) and (b). (a) The maximum possible heights, i.e., the lid, contains one clockwise basic flip labeled in red. (b) The minimum possible heights, i.e., the container, contains nine counterclockwise basic flips labeled in yellow. (c) The 3D shape of a full stack, including a lid (d) and a container (e). (f) The height difference between the lid and the container is a pyramid. (g-j) Upside-down geometries of (c-f). The lid (h) emerges from the maximum packing in the container (i).

in Figs. 6(c) and 6(d). The circular disordered "liquid" region is known as the arctic circle phenomenon [18]. The container shape provides an intuitive understanding about how bound-

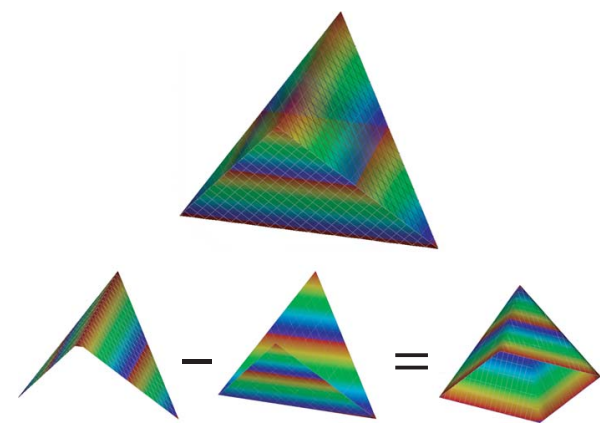

FIG. 5. (Color online) A tetrahedron (top) and its lid, container and their height difference (bottom). 
(A)

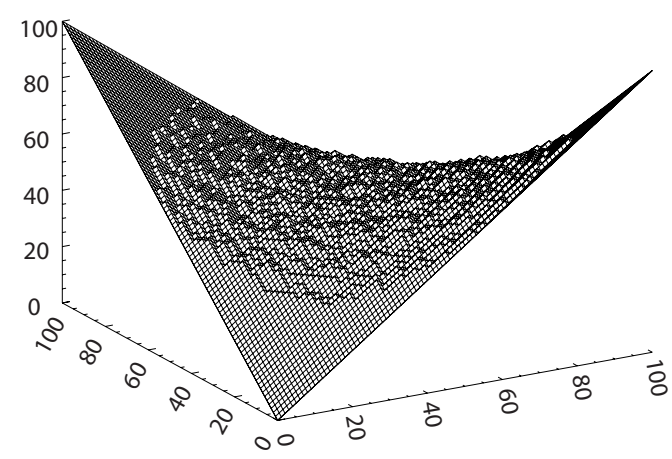

(C)

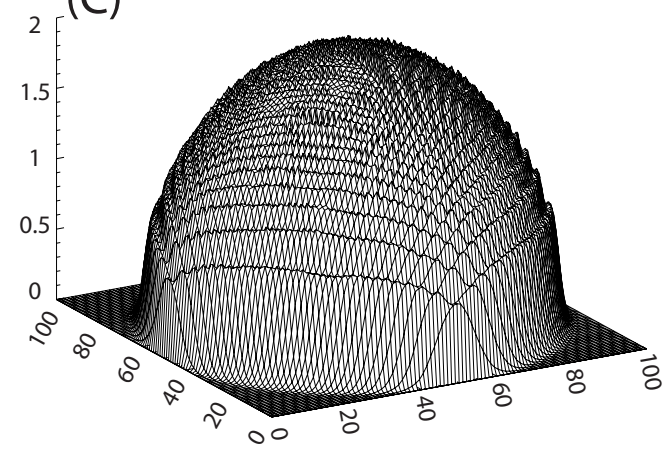

(B)

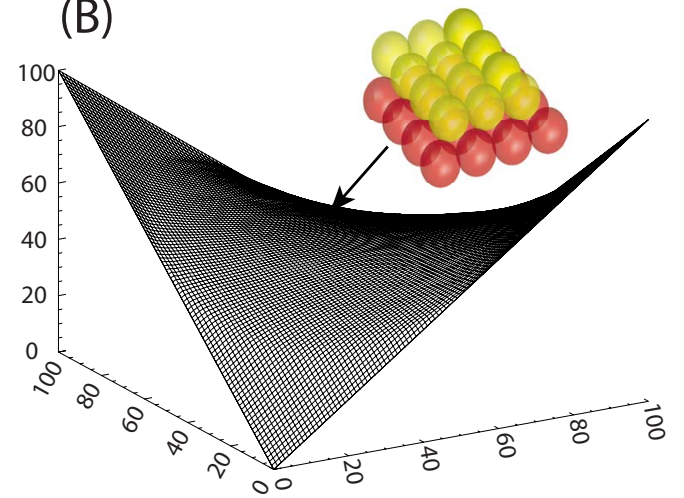

(D)

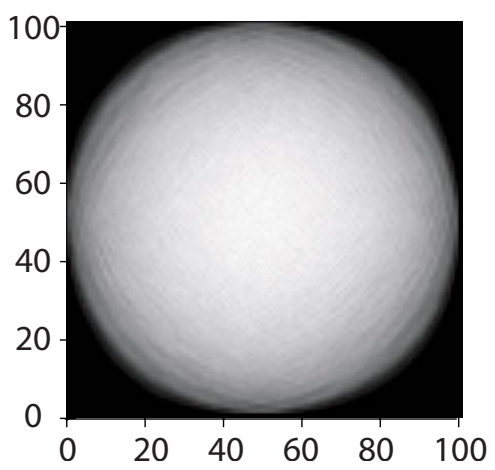

FIG. 6. (Color online) (a) A typical sphere stack in an $L=100$ tetrahedron. The sphere centers are connected so that it appears to be a stack of polyhedra. (b) The mean sphere-stack surface averaged over $10^{9}$ stacks at equilibrium. (b) is the limiting surface of (a) when $L \rightarrow \infty$ [16]. (c) The flipping probability distribution measured from a $10^{9}$-step simulation appears to be a hemisphere. (d) The flipping probability in (c) represented by the brightness.

aries affect the disordered region. In a tetrahedron, the largest horizontal cross section is a square in the middle height that is circumscribed by the disordered region. In an octahedron, however, the largest horizontal cross section is the total area of the $2 \mathrm{D}$ system so that there is no frozen region under the boundary condition shown in Fig. 3. This is confirmed by our simulation. Other boundary conditions may lead to noncircular disordered regions. For example, the flower shape [19] in Fig. 7(b) is a direct consequence of the container shown in Fig. 4(e).

The ensemble average over random stacks results in a mean surface such as the one in Fig. 6(b) or Fig. 7(a). At the infinite-sized limit, dominating number of states are close to
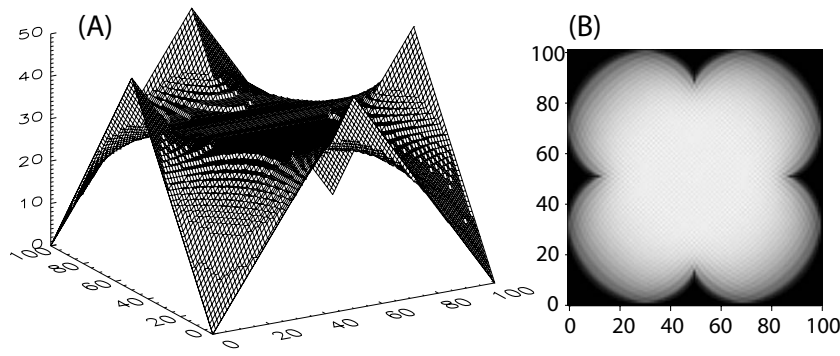

FIG. 7. (a) The ensemble-averaged sphere-stack surface for $L=100$ spin ice with the boundary condition shown in Fig. 4(a). The heights are rounded off to integers in order to show the equalheight contours. The container shape is shown in Fig. 4(e). (b) The flipping probability shown by the brightness. this mean surface [16], i.e., the typical surface in Fig. 6(a) approaches the mean surface in Fig. 6(b) when $L \rightarrow \infty$. The local slope of the surface is related to the density of the basic flips, and the density of the configurational entropy $s_{0}$ [16]. In Fig. 6(b), $s_{0}$ is zero in the frozen areas and continuously increases to reach its maximum value at the center where the local sphere-stack surface is flat. Consequently, the infinitelarge limit under the domain-wall boundary condition cannot be called the thermodynamic limit due to the lack of homogeneity [16]. In contrast, the boundary condition in Fig. 3 has the thermodynamic limit because the limiting surface in the octahedron is flat everywhere, i.e., the density of basic flip is uniform. When the height variation along the fixed boundary is comparable to $L$, the limiting surface is not flat and $\bar{s}_{0}$ is smaller. For example, under the domain-wall boundary condition, $\bar{s}_{0}=k_{B} \ln N_{n}=k_{B} \ln (\sqrt{27 / 16})$ based on Eq. (1), which is smaller than $k_{B} \ln (\sqrt{64 / 27})$ under the periodic boundary condition [6].

\section{NETWORK PROPERTIES}

The phase-space networks can be easily constructed by stacking spheres, see Fig. 8. Two states are connected if they are different by one sphere, i.e., one basic flip. We numerically studied the phase-space networks of the six-vertex models under periodic, free and various fixed boundary conditions. Because the phase space increases exponentially with the lattice size, our computer can only handle the phase 

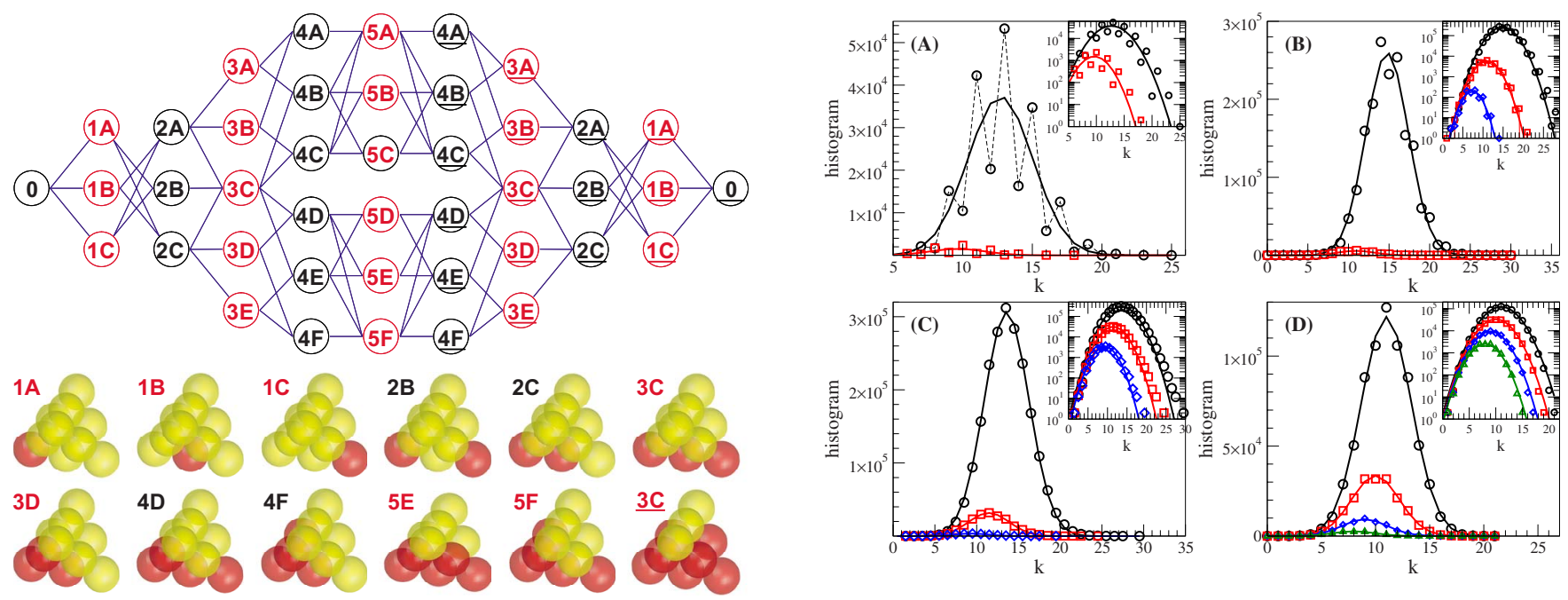

FIG. 8. (Color online) The 42-node phase-space network of the $4 \times 4$ spin ice under the domain-wall boundary condition. The corresponding sphere stacks of some states are shown in the bottom panel. $\underline{3 \mathrm{C}}$ denotes the complementary configuration of $3 \mathrm{C}$.

spaces of small systems. Nevertheless some common features have emerged since the networks are large. These features in the phase spaces of sphere stacks are also shared by the phase-space networks of 2D disk or square stacks shown in Fig. 12.

A fixed boundary condition defines a fixed container shape. Obviously all possible states in a container can be accessed by adding or removing spheres one by one, i.e., the phase space under fixed boundary condition is connected via basic flips as shown in [5].

The distance between two nodes is defined as the shortest path between them. The largest distance in the network, i.e., the network diameter, equals to the number of spheres, which is scaled as $\sim L^{3}$. The numerical results show that the distances between nodes can be scaled onto a binomial-like distribution for various lattice sizes and boundary conditions. Interestingly, the mean distance is roughly proportional to $L$ which increases much more slowly than the network diameter. Consequently the phase spaces have the small-world property [3] since $L$ is logarithmically smaller than the network size, $\sim e^{N_{\text {spin }}} \sim e^{L^{2}}$.

One basic characteristic of networks is the degree (i.e., connectivity) distribution [3]. The connectivity, $k_{i}$, is the number of edges incident with the node $i$. Figure 9 shows the degree distributions of phase-space networks under different boundary conditions. The degree distributions can be well fitted by Gaussian functions, but not by Poisson functions. Hence they are different from the Poisson degree distributions in typical random networks [2,3] and the power-law degree distributions in scale-free networks $[2,3]$. The connectivity $k_{i}$ reflects how frequently the dynamic trajectory visits node $i$. In the network theory, the visiting frequency of a random walker on node $i$ is exactly proportional to its local connectivity $k_{i}[4,20]$. On the other hand, the state with more basic flips (i.e., higher connectivities) can change more easily to other states and result in a shorter staying time. These two effects cancel out so that dynamic trajectories will spend

FIG. 9. (Color online) The degree distributions of the phasespace networks. (a) Sphere stacks in tetrahedra with side length $L=6$ (circles) and $L=5$ (squares), i.e., $7 \times 7$ and $6 \times 6$ spin ices under the domain-wall boundary conditions. (b) $5 \times 5$ (diamonds), $6 \times 6$ (squares) and $7 \times 7$ (circles) spin ices with the boundary conditions shown in Fig. 4(a). (c) $4 \times 3$ (diamonds), $4 \times 4$ (squares) and $4 \times 5$ (circles) spin ices with the free boundary conditions. (d) $2 \mathrm{D}$ disk or square stacks in $L=8$ (triangles), 9 (diamonds), 10 (squares), 11 (circles) container. Insets: semi-log plots. The curves in the main plots and insets show the best Gaussian fits.

equal amounts of time on each node on average and the system is still ergodic, see [4].

An $N_{n}$-node network is described by the $N_{n} \times N_{n}$ adjacent (or connectivity) matrix A. $A_{i j}=1$ if nodes $i$ and $j$ are connected, otherwise $A_{i j}=0$. The adjacent matrixes of phase-

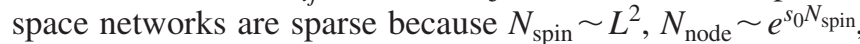
and the highest connectivity $k_{\max } \sim L^{2} \sim N_{\text {spin }} \ll N_{\text {node }}$. Since edges in phase-space networks are undirected, $\mathbf{A}$ is symmetric and all its eigenvalues, $\lambda_{i}$, are real. The eigenvalue spectrum and eigenvectors provide information about their structural properties [21]. For example, if all the $N_{n}$ components of the principle eigenvector are positive, then the network is connected; Otherwise the network has isolated nodes. The principle eigenvalue (i.e., the largest eigenvalue) can be used to identify the community structure [22]. In an uncorrelated random network, the principal eigenvalue $\lambda_{1}$ shows the density of edges and the second largest $\lambda_{2}$ can be related to the diameter and the conductance of the graph as a network resistance [23]. In particular, the spectral density $\rho(\lambda)$ reflects the topology of the network. It is defined as the probability distribution of eigenvalues:

$$
\rho(\lambda)=\frac{1}{N_{n}} \sum_{i=1}^{N_{n}} \delta\left(\lambda-\lambda_{i}\right) .
$$

$\rho(\lambda)$ 's $q$ th moment, $M_{q}$, is directly related to the network's topological feature [21]: $D_{q}=N_{n} M_{q}=\sum_{i=1}^{N}\left(\lambda_{i}\right)^{q}$ is the number of paths (or loops) that return back to the original node after $q$ steps [3]. In sphere-stack networks, all closed paths have even steps so that all odd moments are zero. Consequently, the spectral density is symmetric and centered at zero. The 


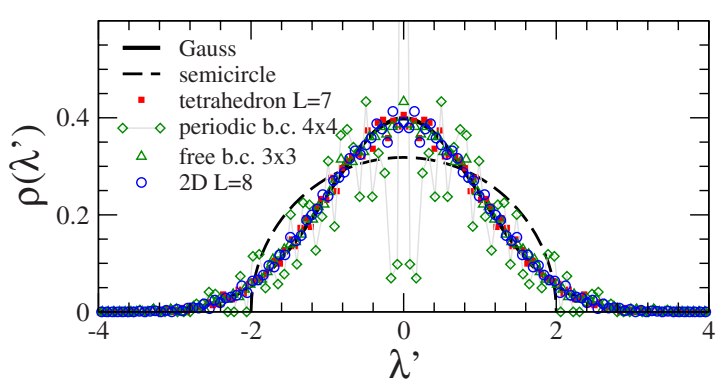

FIG. 10. (Color online) Rescaled spectral densities of phasespace networks. Variances are rescaled to 1 by $\lambda^{\prime}=\lambda / \bar{k}^{\frac{1}{2}}$. Solid squares: the 7436-node network of the $7 \times 7$ lattice with the domain-wall boundary condition, i.e., sphere stacks in $L=6$ tetrahedron; Diamonds: the 2970-node network of $5 \times 5$ lattice with the periodic boundary condition. The data point of $\rho(0)=2.04$ is out of the figure. This peak reflects many starlike and isolated nodes under the periodic boundary condition; triangles: the 2604-node network of $3 \times 3$ lattice with the free boundary condition; circles: the 12870 node network of $8 \times 8$ square stacks in 2D. Black curve: Gaussian distribution $e^{-\lambda^{\prime 2} / 2} / \sqrt{2 \pi}$. Dashed curve: Wigner's semicircle law for random networks. $\rho(\lambda)=\sqrt{4 \sigma^{2}-\lambda^{2}} /\left(2 \pi \sigma^{2}\right)$ if $|\lambda|<2 \sigma$ and zero otherwise. The variance $\sigma^{2}$ is also rescaled to 1 .

$i$ th node with $k_{i}$ neighbors has $k_{i}$ ways to return back after two steps; hence, the variance $\sigma^{2}=M_{2}=\Sigma_{i} k_{i} / N_{n}=\bar{k}$, where $\bar{k}=2 N_{\text {edge }} / N_{n}$ is the mean connectivity. In Fig. 10, we rescaled the measured spectral densities by $\bar{k}^{1 / 2}$ to the unit variance and they all collapsed onto the same Gaussian distribution. Such Gaussian behavior is expected to hold at the largesize limit as long as the model can be mapped as a stack of building blocks, see the proof in [4]. Since the six-vertex models can be mapped as sphere stacks, the proof in [4]. holds and the spectral density become exact Gaussian when the system is large. In practice, the spectral densities are already Gaussian in our small systems. For example, all the Gaussian fits in Fig. 10 are indistinguishable from the $e^{-\lambda^{\prime 2} / 2} / \sqrt{2 \pi}$ curve. In contrast, the spectral density is a semicircle for a random network at large-size limit, a triangular distribution with power-law tails for scale-free networks, and irregular distributions for small-world, modular hierarchical and many real-world networks [21]. Thus, these phase-space networks belong to a new class of complex networks.

\section{DISCONNECTED PHASE SPACES}

Unlike the free and fixed boundary conditions, the periodic boundary conditions in frustrated spin systems lead to disconnected phase spaces [24,25]. Here, we quantitatively calculate the number and the sizes of some disconnected phase-space subnetworks. First we consider the example in Fig. 11, which shows all the 44 configurations of the $2 \times 3$ spin ice with a toroidal boundary condition. Note that this periodic boundary condition is for spins, not for heights. The upper left corner is defined as zero height. The 12 configurations in Fig. 11(a) are connected by basic flips and form a 12-node cluster as shown in Fig. 11(d). The other 12 configurations in Fig. 11(b) form another 12-node cluster in Fig. 11(d). The height difference between the top and the bottom corners is +1 in Fig. 11(a) and -1 in Fig. 11(b). Note that the four corners are essentially the same plaquette on the toroid, so they must be either all inside or all outside of a loop. Consequently, the height differences between the corners cannot be changed by flipping a closed spin loop, see Fig. 11(e). Therefore, the configurations in Figs. 11(a) and 11(b) form two disconnected clusters in Fig. 11(d). The 20 isolated nodes in Fig. 11(d) correspond to the 20 configurations in Fig. 11(c), none of which contains basic flips.

We generalize the above results to the $m \times n$ periodic lattice and prove that it has $(m-1) \times(n-1)$ nontrivial clusters and $2^{n+1}+2^{m+1}-4$ isolated nodes. After walking along the two closed loops along the $x$ and $y$ directions on the toroid and coming back to the original plaquette, the height may change. Such height differences, $\Delta h_{x}$ and $\Delta h_{y}$, uniquely characterize each disconnected subnetwork. We choose the configuration whose bulk spins are along the boundary spins to represent each subnetwork, e.g., configuration 1 in Fig. 11(a), configuration 13 in Fig. 11(b) and all configurations in Fig. 11(c). First, we consider the number of isolated nodes in phase space whose configurations have no basic flips. For such a configuration, all of its horizontal spins or vertical spins have to be along the same direction as shown in Fig. 11(c). If all horizontal spins are leftwards (or rightwards), there are $2^{m}$ configurations for vertical spins [see Fig. 11(c)]. If all vertical spins are upwards (or downwards), there are $2^{n}$ configurations for horizontal spins. Four configurations have both horizontal and vertical parallel spins. These four configurations are double counted so that in total there are $2^{n+1}+2^{m+1}-4$ isolated nodes as shown in Fig. 11(c). Next, we consider the nontrivial clusters with multiple nodes. The corner height difference, $\Delta h_{x}$, has $m-1$ possible values, and $\Delta h_{y}$ has $n-1$ possible values [see Fig. 11(f)], so that there are $(m-1) \times(n-1)$ nontrivial subnetworks in total. The representative configurations of the six subnetworks shown in Fig. 11(f) are chosen to have the lowest possible heights, i.e., vacant containers for sphere stacking. Each configuration is characterized by one basic flip labeled as a yellow square, i.e., the lowest point of the vacant container. Apparently, there are $(m-1) \times(n-1)$ positions for the yellow square, i.e., $(m-1) \times(n-1)$ subnetworks. The subnetwork with the minimum $\Delta h_{x}$ and $\Delta h_{y}$ (i.e., 0 or \pm 1 ) has the largest subnetwork because it corresponds to the constant-height boundary condition. This result implies that the zero-point entropy (i.e., entropy per spin at the ground state) $\bar{s}_{p}$ of the whole network under the periodic boundary condition is the same as the entropy $\bar{s}_{s}$ of the largest subnetwork under the constantheight boundary condition because $(m-1) \times(n-1)$ is logarithmically smaller than $e^{m \cdot n}$ :

$$
\begin{gathered}
\bar{s}_{s}<\bar{s}_{p}=\frac{1}{N_{\text {spin }}} \ln N_{\text {node }}^{p}<\frac{1}{N_{\text {spin }}} \ln \left[(m-1)(n-1) N_{\text {node }}^{s}+2^{m+1}\right. \\
\left.+2^{n+1}-4\right] \simeq \frac{1}{N_{\text {spin }}}\left[\ln (m-1)+\ln (n-1)+\ln e^{\bar{s}_{s} N_{\text {spin }}}\right] \\
\simeq \frac{1}{m n}[\ln (m-1)+\ln (n-1)]+\bar{s}_{s} \simeq \bar{s}_{s}
\end{gathered}
$$


(A)

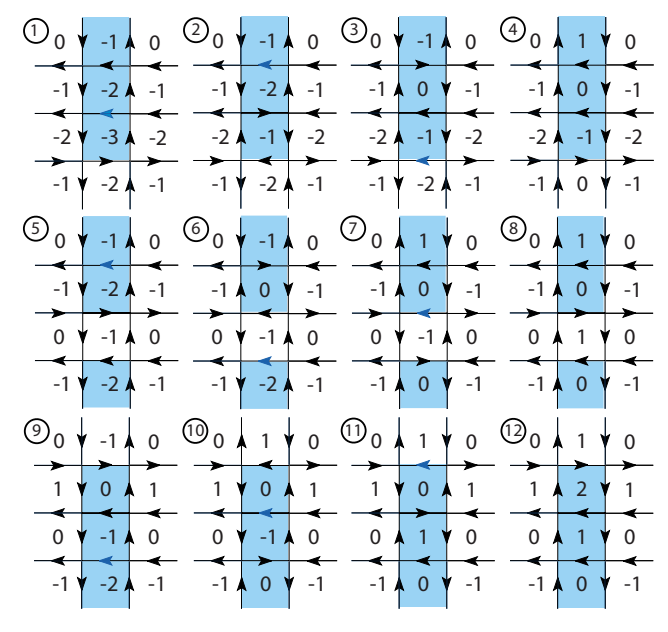

(C)
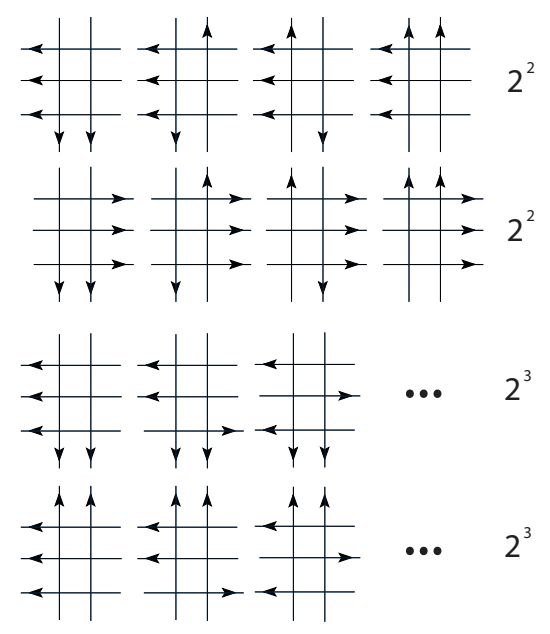

$$
2 \times 2^{2}+2 \times 2^{3}-4=20
$$

(E)

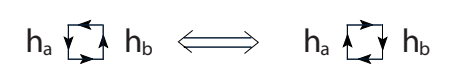

(B)

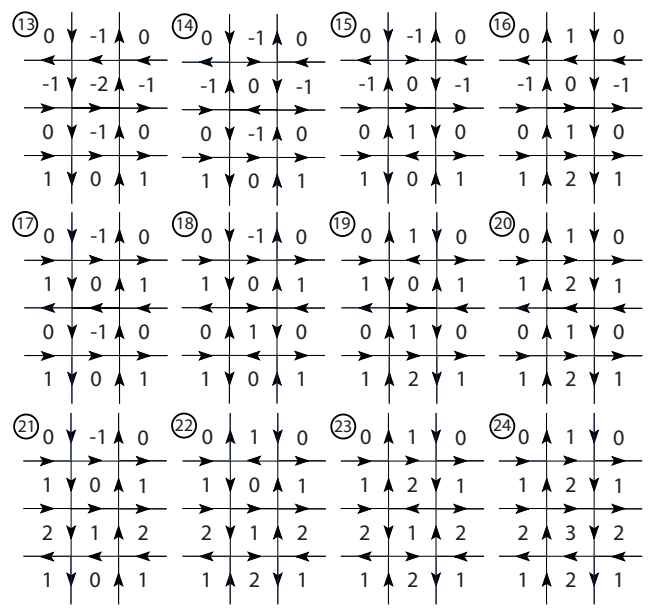

(D)

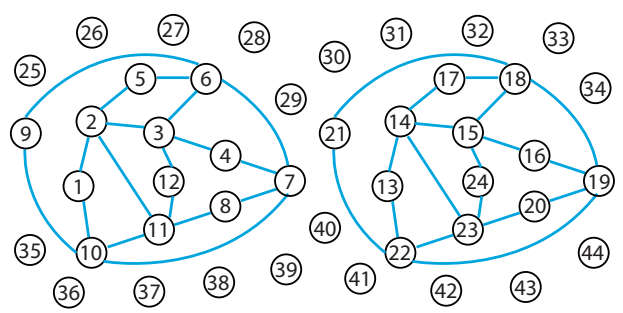

(F)

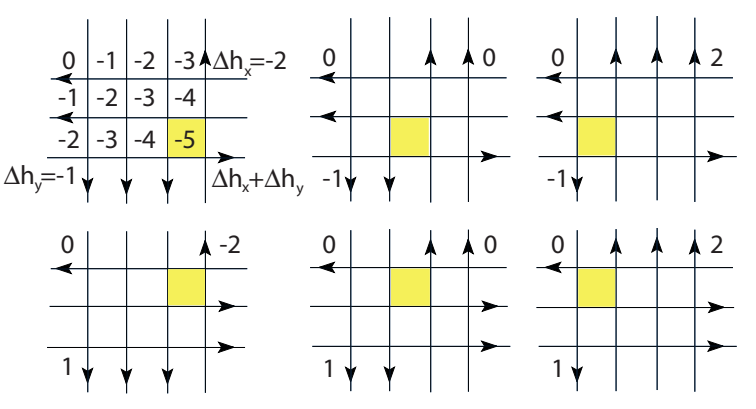

FIG. 11. (Color online) Configurations and the phase-space network of $2 \times 3$ spin ice with the periodic boundary condition. (a) Configurations 1 to 12. The upper left plaquette is defined as zero height and other heights are derived from the height rule in Fig. 1(e). (b) Configurations from 13 to 24 . (c) 20 configurations that contain no basic flips. They are categorized into four types: all horizontal spins are (1) leftwards; (2) rightwards; all vertical spins are (3) upwards; (4) downwards. Four configurations are double counted, so the total number of configurations is $2 \times 2^{2}+2 \times 2^{3}-4=20$. (d) The whole phase-space network with 44 nodes. (e) Flipping a loop does not change the height difference if two plaquettes are both inside or outside the loop. (f) $3 \times 4$ spin ice with periodic boundary conditions. There are $(3-1) \times(4-1)=6$ types of different $\left(\Delta h_{x}, \Delta h_{y}\right)$, i.e., 6 nontrivial subnetworks. The 6 representative configurations for the 6 subnetworks have the lowest heights which define their container shapes. Each container has a unique lowest height labeled in yellow.

Next, we show that the smallest nontrivial cluster has $\frac{(m+n-1) !}{(n-1) !(m-1) !}$ nodes. In Fig. 11(f), the two middle configurations are the representative states for the two 132-node subnetworks and the other four configurations are the representative states for the four 60-node subnetworks. These four configurations with the lowest heights at the corners define tilted 2D containers rather than 3D containers, for example see the first panel of Fig. 11(f). Thus, the number of spheres packed in such a container is much smaller than that in $3 \mathrm{D}$ containers whose lowest point (the yellow plaquette) is not at the corner. To count the number of stacks in a tilted 2D container, we first consider a simple example in Fig. 11(a). Configuration 1 in Fig. 11(a) is the representative state of the subnet- work with the lowest height of -3 . Configurations 1 to 4 in Fig. 11(a) have the same boundary spins so that we can view them as the $2 \mathrm{D}$ sphere stacks in the same $1 \times 3$-sized $2 \mathrm{D}$ rectangle. Such a blue $1 \times 3$ container has three possible positions relative to the zero height plaquette, see configurations 1, 5, and 9 in Fig. 11(a). Hence the subnetwork has 3 $\times 4=12$ nodes in total. It is easy to generalize this counting to $m \times n$ square ice on a toroid. There are $m$ possible positions for the $m \times(n-1)$-sized rectangle. For 2D sphere stacking in an $m \times(n-1)$ container, there are $C_{m+n-1}^{m}$ configurations, see Fig. 12 and its caption. Consequently, there are $m C_{m+n-1}^{m}=\frac{(m+n-1) !}{(n-1) !(m-1) !}$ nodes in the smallest nontrivial subnetworks. All the above formulas are confirmed by our numeri- 


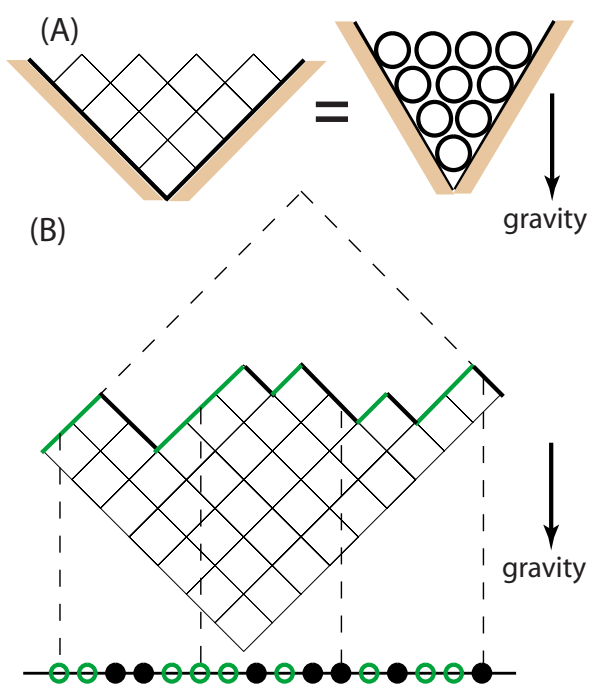

FIG. 12. (Color online) (a) The 2D circle stacks have one-to-one correspondence to the 2D square stacks. Each circle/square is supported by two circles/squares underneath in a gravity field. (b) Mapping a $2 \mathrm{D}$ stack of squares in an $a \times b=7 \times 9$ container to a chain of $a$ solid particles and $b$ holes [26]. The dynamics of adding/removing squares can be described as the diffusion of particles in a symmetric simple exclusion process (SSEP). The number of $2 \mathrm{D}$ stack configurations in a container is $C_{a+b}^{a}=(a+b) ! /(a ! b !)$, i.e., the number of ways to put $a$ particles onto $a+b$ sites.

cal results at $m, n<8$. Our results at $m=n$ agree with the number sequence A054759 in [27] for the $n \times n$ spin ice under the periodic boundary conditions.

The phase-space subnetworks of the periodic boundary conditions share the same properties of the phase-space networks under fixed boundary conditions. For example, the phase space of the $4 \times 5$ lattice under the periodic boundary condition consists of 12 disconnected nontrivial subnetworks and 92 trivial isolated nodes. Four identical nontrivial subnetworks have 280 nodes each, two have 730 Nodes each, four have 1280 nodes each and two have 4550 nodes each. The four types of the subnetworks all have Gaussian spectral densities, see Fig. 13. The peaks at zero reflect the star-like structures in the networks [28].

\section{SUMMARY AND OUTLOOK}

We constructed a one-to-one mapping between the sixvertex model and the stack of close-packed spheres. Different boundary conditions correspond to different container shapes. The container and the lid form a pair of dual surfaces. The container shape can qualitative elucidate the boundary effect.

Sphere stacking is not only helpful to phase-space network construction, but also interesting in combinatorics in its own right. Sphere packing is a branch of discrete mathematics. However a typical sphere-packing problem is to find the arrangement that fills the space most efficiently, not their combinatoric properties in a container. Currently the only available combinatoric formula for sphere packing is Eq. (1), which gives the number of alternating signed matrixes. Since

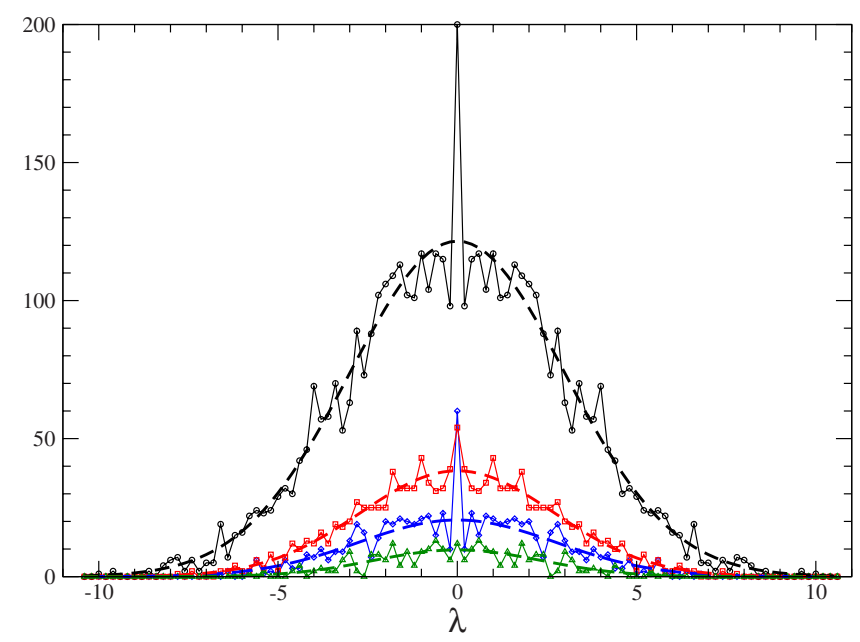

FIG. 13. (Color online) The eigenvalue histograms of the four disconnected subnetworks of the phase space of $4 \times 5$ lattice under the periodic boundary condition. The four subnetworks have 280, 730,1280 , and 4550 nodes, respectively.

alternating signed matrixes and sphere stacks in a tetrahedron have one-to-one correspondence, Eq. (1) also gives the number of ways to pack spheres in tetrahedra. We numerically verified that there are $2,7,42,429,7436,218348 \cdots$ (i.e., the sequence A005130 in [27]) ways to pack spheres in $L=1,2,3,4,5,6, \cdots$ tetrahedra. We also numerically obtained $2,18,868,230274, \cdots$ ways to pack spheres in $L$ $=1,2,3,4, \cdots$ octahedra, but a quantitative formula for this number sequence is not available.

The combinatoric properties of sphere stacking can be similarly studied as the cube stacking. The cube stacking is not only equivalent to the random rhombus tiling or the plane partition in pure mathematics [10], but also naturally appears in many chemical and physical problems such as counting benzenoid hydrocarbons, percolation, crystal melting and string theory [29]. In particular, it has a one-to-one correspondence to the frustrated antiferromagnetic Ising spins on $2 \mathrm{D}$ triangular lattice $[4,8]$. In contrast to the intensively studied cube stacking, sphere stacking has not been explored. Many questions studied in cube stacking can be asked about sphere stacking. For example, how many ways are there to pack $N$ spheres into a tetrahedron? What are the analytical forms of the limiting surfaces in Fig. 6(a), i.e., what are the entropy density distributions [16,30]? These questions can also be asked about other container shapes such as octahedron. In particular We measured the number of packing configurations $W(n)$ as a function of the number of "sand grains" $n$. Interestingly we found that $W(n)$ of different sand grains (such as 2D squares, 3D cubes, 3D spheres) and different containers exhibit the same limiting behaviors, which suggests some fundamental connections between the generating functions of these systems. Note, the generating functions $[10,31]$ of sphere stacks in different containers are still open questions.

Moreover, the six-vertex model has one-to-one correspondences to other 2D models, such as gog triangles [32] threecolor graphs, dimers, fully packed loops, etc [33]. It also has a one-to-multiple correspondence to the domino tiling [34]. 
Sphere stacking is a new equivalent representation in $3 \mathrm{D}$ with more transparent symmetries. Hence it may cast new lights on these 2D models, in particular, on the conjecture of the one-to-one mapping between gog triangles and magog triangles [32].

We mapped out the phase-space networks of small sixvertex models under different boundary conditions. In contrast to some conventional phase-space studies which used a random walker to explore a small portion of a huge phase space, we mapped out the whole phase spaces so that exact results can be obtained from quantitative network analysis. Although we can only measure phase-space networks of small systems, these numerical results have allowed us to show that the observed features also hold in large systems. We observed that the phase-space networks have the smallworld property, the Gaussian-like connectivity distributions and the Gaussian spectral densities. These features are also shared by the ground-state phase spaces of frustrated antiferromagnets on triangular lattices (see [4]) and the phase-space networks of the particles diffusion in one-dimensional lattice (i.e., the 2D square stacking shown in Fig. 12). It would be interesting to explore whether these features universally hold in phase spaces of other frustrated spin models and particlediffusion models. In particular, the Gaussian spectral densities reflect the unique network structures and distinguish the phase spaces from social networks, information networks, biological networks and technological networks [2]. We also derived a few formulas about the number and the sizes of some disconnected phase-space subnetworks under periodic boundary conditions. The sizes of other subnetworks are still challenging.

The connections between the frustrated spin model and complex networks give rise to new open questions. For example, how do we calculate the number of edges in the phase-space network? How do we prove that the degree distributions in Fig. 9 are Gaussian? Is the flipping probability in Fig. 6(c) a hemisphere? Are there any highly connected communities in the phase spaces? Do phase spaces have fractal structures? Some of these questions can be directly answered by the complex network analysis developed in the recent decade [3]. A number of network analysis techniques have been readily applied to the phase-space study, such as the correlations [3], centrality [3], community structures [22], fractal properties [35] and coarse graining [36]. These techniques can provide a better understanding of the phasespace structures.

In particular, the network community analysis may provide a mean to quantify the weak ergodicity. A network is said to have community structure if it divides naturally into groups of nodes with dense connections within groups and sparser connections between groups. If the phase-space network have strong community structure, the system will be trapped in a small part of its phase space for a very long time, which is usually called as weak ergodicity or entropic barrier [37]. Network analysis can detect the community structures if there are any, and the "strength" of each community is characterized by the modularity [22]. This provides a quantitative measure for the weak ergodicity, which was not available before. Moreover, the network fractal analysis may cast lights on the highly controversial nonextensive Tsallis entropy $[38,39]$, which is a generalization of Boltzmann entropy for nonequilibrium or long-ranged interacting systems. Tsallis entropy is based on the assumption that the long-ranged interacting systems have fractal phase spaces [38]. However, a real example of fractal phase space was not available yet. In the six-vertex model, the boundary effect can percolate through the entire system and exhibit longrange interacting features. Hence, it would be interesting to explore whether the phase spaces are fractal by using the network fractal analysis [35].

\section{ACKNOWLEDGMENT}

We thank Michael Wong for helpful discussions.
[1] R. Albert and A.-L. Barabási, Rev. Mod. Phys. 74, 47 (2002).

[2] M. E. J. Newman, SIAM Rev. 45, 167 (2003).

[3] L. D. F. Costa, F. A. Rodrigues, G. Travieso, and P. R. Boas, Adv. Phys. 56, 167 (2007).

[4] Y. Han, Phys. Rev. E 80, 051102 (2009).

[5] K. Eloranta, J. Stat. Phys. 96, 1091 (1999).

[6] E. Lieb, Phys. Rev. Lett. 18, 692 (1967); Phys. Rev. 162, 162 (1967).

[7] G. H. Wannier, Phys. Rev. 79, 357 (1950); Phys. Rev. B 7, 5017 (1973) erratum.

[8] H. W. J. Blöte and B. Nienhuis, Phys. Rev. Lett. 72, 1372 (1994).

[9] Y. Han et al., Nature (London) 456, 898 (2008).

[10] D. M. Bressoud, Proofs and Confirmations: The Story of the Alternating-Sign Matrix Conjecture (Cambridge University Press, Cambridge, England, 1999).

[11] H. van Beijeren, Phys. Rev. Lett. 38, 993 (1977).

[12] M. T. Batchelor, R. J. Baxter, M. J. O'Rourke, and C. M.
Yung, J. Phys. A 28, 2759 (1995)

[13] V. E. Korepin, Commun. Math. Phys. 86, 391 (1982).

[14] R. J. Baxter, Exactly Solved Models in Statistical Mechanics (Academic Press, London, 1982).

[15] R. P. Millane and N. D. Blakeley, Phys. Rev. E 70, 057101 (2004).

[16] N. Destainville, J. Phys. A 31, 6123 (1998).

[17] P. Zinn-Justin, e-print arXiv:cond-mat/0205192.

[18] W. Jockush, J. Propp, and P. Shor, e-print arXiv:math/ 9801068.

[19] K. Eloranta, e-print arXiv:0909.4007.

[20] J. D. Noh and H. Rieger, Phys. Rev. Lett. 92, 118701 (2004).

[21] I. J. Farkas, I. Derényi, A.-L. Barabási, and T. Vicsek, Phys. Rev. E 64, 026704 (2001); M. A. M. de Aguiar and Y. BarYam, ibid. 71, 016106 (2005).

[22] M. E. J. Newman, Proc. Natl. Acad. Sci. U.S.A. 103, 8577 (2006).

[23] Handbook of Combinatorics, edited by R. L. Graham, M. 
Grötschel, and L. Lovász (North-Holland, Amsterdam, 1995).

[24] C. Moore and M. E. J. Newman, J. Stat. Phys. 99, 629 (2000).

[25] T.-K. Ng, Y. Zhou, and L.-H. Tang, EPL 86, 10003 (2009).

[26] A. Okounkov, e-print arXiv:math-ph/0309015.

[27] http://www.research.att.com/ njas/sequences/.

[28] S. N. Dorogovtsev, A. V. Goltsev, J. F. F. Mendes, and A. N. Samukhin, Phys. Rev. E 68, 046109 (2003).

[29] A. Okounkov, N. Reshetikhin, and C. Vafa, Progr. Math. 244, 597 (2006).

[30] R. Kenyon and A. Okounkov, Acta Math. 199, 263 (2007).

[31] G. E. Andrews, Percy Alexander MacMahon: Collected Papers (The MIT Press, Cambridge, 1986), Vol. 2.

[32] D. Bressoud and J. Propp, Not. Am. Math. Soc. 46, 637 (1999).

[33] J. Propp, Discrete Math. Theoret. Comput. Sci. Proc. AA, 43
(2001) [http://www.dmtcs.org/proceedings/html/dmAA0103. abs.html].

[34] N. Elkies, G. Kuperberg, M. Larsen, and J. Propp, J. Algeb. Geom. 1, 111 (1992).

[35] C. Song, S. Havlin, and H. A. Makse, Nature (London) 433, 392 (2005).

[36] D. Gfeller and P. De Los Rios, Phys. Rev. Lett. 99, 038701 (2007).

[37] N. Destainville and V. Desoutter, Appl. Math. Inf. Sci. 2, 83 (2008);V. Desoutter and N. Destainville, J. Phys. A: Math. Gen. 38, 17 (2005).

[38] A. Cho, Science 297, 1268 (2002).

[39] C. Tsallis, Introduction to Nonextensive Statistical Mechanics: Approaching a Complex World (Springer, New York, 2009). 